\title{
SUPPORTING HUMAN-CENTERED DESIGN IN PSYCHOLOGICALLY DISTANT PROBLEM DOMAINS: THE DESIGN FOR CYBERSECURITY CARDS
}

\author{
Rao, Vivek (1); \\ Moore, George (1); \\ Jung, Hyun Jie (1); \\ Kim, Euiyoung (2); \\ Agogino, Alice (1); \\ Goucher-Lambert, Kosa (1) \\ 1: University of California, Berkeley; \\ 2: Delft University of Technology
}

\begin{abstract}
Increasingly digital products and services make cybersecurity a crucial issue for designers. However, human-centered designers struggle to consider it in their work, partially a consequence of the high psychological distance between designers and cybersecurity. In this work, we build on the Design for Cybersecurity (DfC) Cards, an intervention to help designers consider cybersecurity, and examine a project-based design course to understand how and why specific DfC cards were used. Three findings result. First, designers found the intervention useful across all design phases and activities. Second, the cards helped design teams refocus their attention on the problem domain and project outcome. Third, we identify a need for support in framing and converging during user research, opportunity identification, and prototyping. We argue that the psychological distance between designers and the problem space of cybersecurity partially explains these findings, and ultimately exacerbates existing challenges in the design process. These findings suggest that design interventions must consider the psychological distance between designer and problem space, and have application in design practice across many complex problem domains.
\end{abstract}

Keywords: Creativity, Human behaviour in design, Design cognition, Cybersecurity

\section{Contact:}

Rao, Vivek

UC Berkeley

Mechanical Engineering

United States of America

vivek.rao@berkeley.edu

Cite this article: Rao, V., Moore, G., Jung, H. J., Kim, E., Agogino, A., Goucher-Lambert, K. (2021) 'Supporting Human-Centered Design in Psychologically Distant Problem Domains: The Design for Cybersecurity Cards', in Proceedings of the International Conference on Engineering Design (ICED21), Gothenburg, Sweden, 16-20 August 2021. DOI:10.1017/pds.2021.544 


\section{INTRODUCTION}

Designers today must address complex challenges that accompany common products, services, and systems (Norman and Stappers, 2015). Notable among these challenges is cybersecurity, critically important to increasingly connected products, services, and systems (TwenSeboah-Koduah et al., 2017). However, cybersecurity is often not seen as an 'essential design principle' (Schwartz et al., 2018), and research suggests that cybersecurity awareness across the design process is inconsistent (Kim et al., 2018). Efforts to incorporate cybersecurity in the design process have focused on software (Assal, 2018; Lukowiak et al., 2014), but less attention has been paid to early-stage conceptual design, and specifically, human-centered design (HCD).

$\mathrm{HCD}$, often described with phases of Research, Analyze, Ideate, Build, and Communicate (Roschuni et al., 2011), intends to help designers tackle complex problems, including cybersecurity. While HCD approaches promise to help designers establish empathy and understanding for such complex problem spaces, as is well-documented in health (Bazzano et al., 2017), achieving empathetic outcomes remains challenging (Postma et al., 2012).

One driver of this challenge is the psychological distance between a designer and a complex problem. Psychological distance, as described by Trope and Liberman's Construal Level Theory (CLT), is "a subjective experience that something is close or far away from the self, here, and now" (Trope and Liberman, 2010). High psychological distance makes a problem more abstract (Trope and Liberman, 2010), and thus, difficult to design for. In HCD, empathizing with users is intended to bridge psychological distance (Liberman and Trope, 2008), which has been shown to increase task engagement (Hoever et al., 2012; Pahl and Bauer, 2013). However, bridging this distance when the problem in question is a complex sociotechnical challenge, like cybersecurity, is especially challenging - and can cause psychological distance to persist even in HCD. Thus, beyond design process challenges, designers tackling sociotechnical problems like cybersecurity must also bridge the high psychological distance to aspects of their problem that may be adjacent, but essential, to end-use case.

One intervention to help designers engage with cybersecurity during HCD is the Design for Cybersecurity (DfC) card set (Rao et al., 2020a), which seeks to leverage design creativity stimuli to help designers consider cybersecurity in early-stage conceptual design. The cards intend to support creativity across discrete HCD activities and creativity modes and have been shown to be useful in generic design contexts (e.g., not cybersecurity-specific). Three design cards exist for each HCD phase for a total of 15 cards. Each card consists of a stimulus question based on one of three design creativity modes underpinning activities in the design process: analysis, generation, and evaluation (Howard et al., 2008; Rao et al., 2020a). Analysis stimuli help synthesize information to establish constraints. Generation stimuli elicit creative and uninhibited exploration. Evaluation stimuli inspire objective reflection about the team's progress.

In this work, we study the cards' impact as novice designers use them across the HCD process in a project-based learning experience. We extend previous work to explore how domain-specific creativitybased interventions support designers across HCD phases when projects are (1) cybersecurity-specific and (2) psychologically distant. Under these conditions, our research questions are:

- R1: How do designers perceive the utility of the cards across design phases?

- R2: How do designers' usages of the cards align with the intent of the cards?

To explore these questions, we follow six student design teams across the HCD process, deploying the DfC cards and surveying students to understand how and why they seek support in cybersecurity design projects. The main contributions of these work are (1) longitudinal insights across the HCD process about how designers leverage domain-specific design creativity stimuli when working with psychologically distant problem domains; (2) identification of particular process-oriented challenges HCD teams face when engaging with psychologically distant problem domains, like cybersecurity.

We first review key related work on psychological distance and design for cybersecurity (Sec. 2), and then introduce our methodological approach, detailing the project context, the DfC cards, and survey instruments (Sec. 3). Next, we present five results that address our research questions above and discuss their implications for design research and practice (Sec. 4). We close with a discussion of limitations (Sec. 5) and conclusions (Sec. 6) of the work. 


\section{BACKGROUND \& RELATED WORK}

\subsection{Psychological distance and creativity}

In Trope and Liberman's Construal Level Theory (CLT), psychological distance is described via four dimensions: time, space, social distance, and hypotheticality. CLT has been used to explain why individuals respond, or do not respond, to a range of issues, from climate change (Spence et al., 2012) to product pricing (Bornemann and Homburg, 2011). Trope and Liberman argue that the greater the psychological distance an individual has from an object, the more abstract the level of construal of that object the individual has (Trope and Liberman, 2010). In the case of cybersecurity, this abstraction has been used to explain why many efforts to change behavior around data privacy have failed (Schuetz et al., 2015). Cybersecurity appears typically distal by the four CLT measures: it exists far away in time, exists in digital rather than physical space, often does not have cultural or social relation to a user, and unless an individual has been hacked, has high hypotheticality. Combined, these factors make cybersecurity psychologically distant for many individuals.

From a designer's perspective, however, studies have found that psychological distance has a complex relationship with creativity and design outcomes. Polman and Emich found that ideating for others (high social distance) produced more creative solutions than ideating for the self (Polman and Emich, 2011). Jia et al. showed that when creative tasks are presented as emerging from a far location (high spatial distance), more creative solutions resulted (Jia et al., 2009). Foerster et al. demonstrated that when participants imagined engaging with a task one year out, their creative solutions were improved over when they envisioned engaging with a task one day away. The authors noted while abstract creativity was improved by temporal separation, analytical problem solving suffered (Förster et al., 2004). One recent study explored how a proxy for psychological distance, contextual experience, affected engineering designers' novelty, quantity, and quality of solutions to a specific engineering design task. In this work, Hu and Reid discovered that lack of contextual experience (high psychological distance) produced more novel solutions. More contextual experience (low psychological distance), however, yielded higher quality solutions (Hu and Reid, 2018). However, little is known about how design creativity stimuli perform and are utilized in complex sociotechnical problems that address typically psychologically-distant problem domains.

In our work, we extend on previous studies by examining designers' responses to domain-specific stimuli across the HCD process when projects are psychologically distant. Rather than examine a specific task, we study the many tasks involved in HCD, from user research to prototyping. By focusing on cybersecurity-related projects, we explore how domain-specific stimuli help designers navigate psychologically distant problem sets. This effort is intended to help designers maintain design creativity in high psychological distance environments, as balancing Hu and Reid's tradeoff between quality vs. novelty of ideas is crucially important to successfully supporting design teams.

\subsection{Cybersecurity and conceptual design}

The challenges, nuances, and complexities of delivering cybersecurity outcomes have yielded a welldocumented nationwide shortage in cybersecurity talent, which has the potential to affect all sectors of the economy and government (Crumpler and Lewis, 2019). Efforts to help software designers and engineers incorporate cybersecurity (Lukowiak et al., 2014) typically focus software engineering, and often do not sufficiently scale to early-stage conceptual engineering design challenges.

Efforts to make cybersecurity accessible to designers have focused on making cybersecurity tangible. Kim's work illustrated the limitations of this approach, however: while such interventions yielded a boost in cybersecurity awareness early in the design process, increases were difficult to sustain over the length of the design process (|Kim et al., 2019, 2018). Tangibility seeks to effectively bridge psychological distance between the subject and the issue at hand, allowing designers to engage more concretely with an otherwise-abstract challenge area. Specific interventions include mixed reality simulations, board games, and card decks, covered in detail elsewhere (Rao et al., 2020a).

Our work expands on Kim's contribution by studying why interventions to support cybersecurity in early-stage conceptual design are effective, grounded in the Design for Cybersecurity cards. Previous work suggests that the Design for Cybersecurity Cards are perceived as having high utility across the entire design process, independent of project type and design phase (Rao et al., 2020a). In this work, we explore beyond perceived utility to understand why and how designers leverage these interventions. 


\section{METHODS}

This study was performed at a major public research university in the United States with a pool of 22 students, with $50 \%$ of students identifying as male and 50\% identifying as female. The majority of students were from the United States (95\% United States-based / 5\% non-United States-based). The largest single discipline represented was engineering (6 students) with other students representing business, chemistry, design, and other majors. All course activities were conducted remotely.

The course focused on using Human Centered-Design as a framework to explore solutions within the context of cybersecurity. Project topics were pre-defined by course faculty, and six student teams were formed to explore different cybersecurity-specific topics, ranging from diagnosing advanced persistent threats in network security to building end-user awareness about smart home device privacy and security challenges. The course was guided students through five design phases of the HCD process (Research, Analyze, Ideate, Build, and Communicate), and required several final project deliverables: a digital prototype, a physical prototype, a video illustrating the value of the prototype, and a project presentation. By discretely separating core HCD activities in the course, we sequenced the delivery of our interventions to precisely accompany specific design tasks.

Design for Cybersecurity (DfC) Cards (Fig. 1) were used as an intervention to facilitate the work of student teams during each design phase. As described in Section 1, each card provides a stimulus question (Table 3 ) to inspire the team to examine cybersecurity alongside each design phase's goal. Each team used Mural (www.mural.co) to document their progress through each design phase. During each design phase, the three cards from that phase, as illustrated in Figure 1, were presented to students directly in their Mural workspace adjacent to various design tasks.

(A)

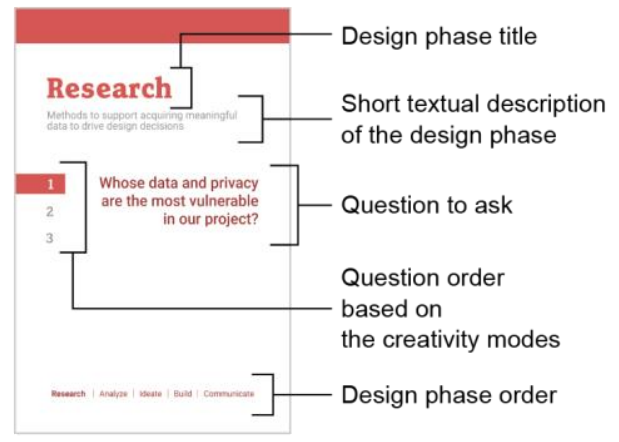

(C)

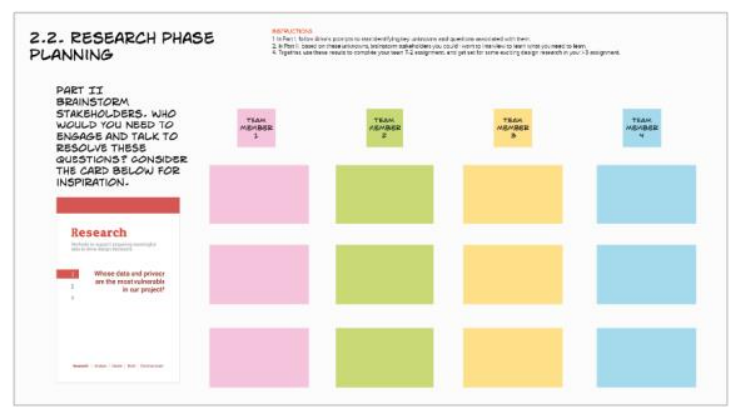

(B)

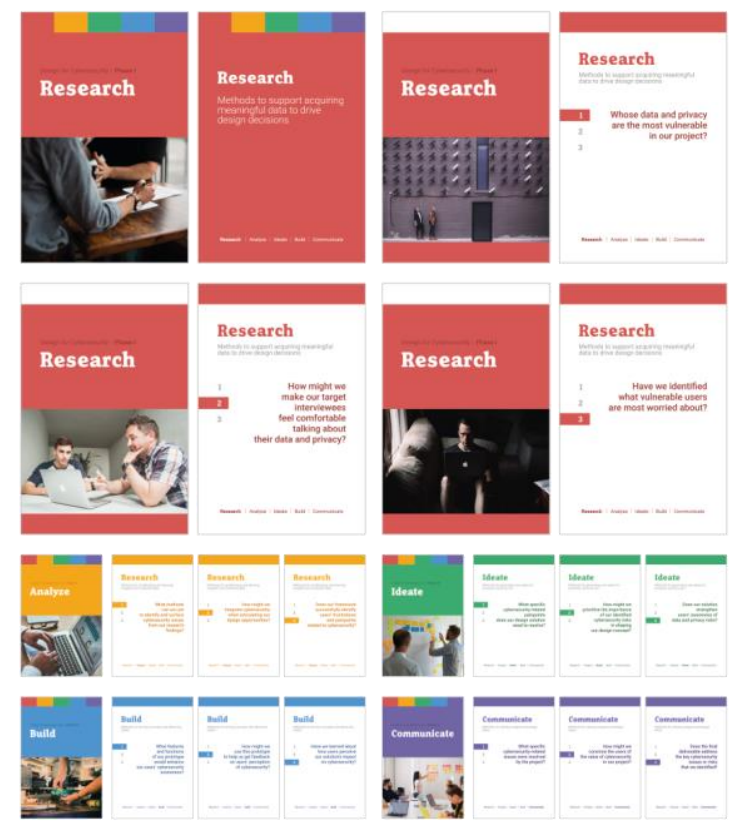

Figure 1. Card Design (A), Collection (B), and integration with Mural platform (C).

At the end of each phase, students were asked (1) how they perceived the utility of the cards on a 1-5 scale, and asked to explain why; (2) how they perceived the cards' value for design activities essential to all phases of the design process, specifically: (1) framing what they needed to do (frame), (2) creating a range of options of what they could do (diverge), or (3) choosing what options they ought to pursue (converge). For each, students could identify more than one card or none of the cards, as valuable. Based on each creativity mode, a specific design activity was intended to be supported. Across all phases, Card 1 represented the analysis creativity mode supporting frame activities; Card 2 represented the generation creativity mode, supporting diverge activities; and Card 3 represented the evaluation creativity mode, supporting converge activities (Table 1). 
Table 1. Card content by phase and creativity mode.

\begin{tabular}{|c|c|c|c|}
\hline \begin{tabular}{|l} 
Design \\
Phase
\end{tabular} & \begin{tabular}{|l} 
Creativity \\
Mode
\end{tabular} & \begin{tabular}{|l|} 
Design \\
Activity
\end{tabular} & Stimulus Question \\
\hline Research & Analysis & Frame & $\begin{array}{l}\text { Whose data and privacy are the most vulnerable in our } \\
\text { project? }\end{array}$ \\
\hline Research & Generation & Diverge & $\begin{array}{l}\text { How might we make our target interviewees feel comfortable } \\
\text { talking about their data and privacy? }\end{array}$ \\
\hline Research & Evaluation & Converge & $\begin{array}{l}\text { Have we identified what vulnerable users are most worried } \\
\text { about? }\end{array}$ \\
\hline Analyze & Analysis & Frame & $\begin{array}{l}\text { What methods can we use to identify and surface } \\
\text { cybersecurity issues from our research findings? }\end{array}$ \\
\hline Analyze & Generation & Diverge & $\begin{array}{l}\text { How might we integrate cybersecurity when articulating our } \\
\text { design opportunities? }\end{array}$ \\
\hline Analyze & Evaluation & Converge & $\begin{array}{l}\text { Does our framework successfully identify users' frustrations } \\
\text { and pain points related to cybersecurity? }\end{array}$ \\
\hline Ideate & Analysis & Frame & $\begin{array}{l}\text { What specific cybersecurity-related pain points do our design } \\
\text { solution needs to resolve? }\end{array}$ \\
\hline Ideate & Generation & Diverge & $\begin{array}{l}\text { How might we prioritize the importance of our identified } \\
\text { cybersecurity risks in shaping our design concept? }\end{array}$ \\
\hline Ideate & Evaluation & Converge & $\begin{array}{l}\text { Does our solution strengthen users' awareness of data and } \\
\text { privacy risks? }\end{array}$ \\
\hline Build & Analysis & Frame & $\begin{array}{l}\text { What features and functions of our prototype would enhance } \\
\text { our users' cybersecurity awareness? }\end{array}$ \\
\hline Build & Generation & Diverge & $\begin{array}{l}\text { How might we use this prototype to help us get feedback on } \\
\text { users' perception of cybersecurity? }\end{array}$ \\
\hline Build & Evaluation & Converge & $\begin{array}{l}\text { Have we learned about how users perceive our solution's } \\
\text { impact on cybersecurity? }\end{array}$ \\
\hline Communicate & Analysis & Frame & $\begin{array}{l}\text { What specific cybersecurity-related issues were resolved by } \\
\text { the project? }\end{array}$ \\
\hline Communicate & Generation & Diverge & $\begin{array}{l}\text { How might we convince the users of the value of } \\
\text { cybersecurity in our project? }\end{array}$ \\
\hline Communicate & Evaluation & Converge & $\begin{array}{l}\text { Does the final deliverable address the key cybersecurity issues } \\
\text { or risks that we identified? }\end{array}$ \\
\hline
\end{tabular}

\section{RESULTS \& DISCUSSION}

\subsection{R1: How do designers perceive the utility of the cards across design phases?}

\subsubsection{Result 1.1: The cards were perceived as having high utility across all design phases.}

To examine how designers perceive the utility of the cards, we examined quantitative responses, receiving 279 evaluations of card utility, with a mean of 18.6 responses per card. The cards were perceived as having high overall utility, with a mean of 3.97 on a 5-point scale (sd $=0.97)$. No significant differences between the mean perceived utility of the cards by design phase or by creativity mode were found using a single-factor ANOVA $(\mathrm{p}>0.05)$.

\subsubsection{Result 1.2: The Communicate phase appears to provide the greatest perceived utility.}

We are able to validate the cards' wide perceived utility across design phase and creativity mode. While statistically insignificant, we note that the Communicate phase exhibited the highest mean perceived utility among design phases (4.17), with a narrower standard deviation (0.795). This result reinforces previous findings of high perceived utility across the design process (Rao et al., 2020a). This is especially notable, given the focused nature of student projects on cybersecurity and the fact that the 
class was conducted remotely. Examining why the Communicate phase was the subject of high perceived utility, designers' comments revealed important insight:

"... I found all of these cards helpful for the communication phase. With communication, it is incredibly important to address concerns and needs within our project topic when presenting our final prototypes. The questions of asking what specific cybersecurity issues were resolved and how we could convince users the value of cybersecurity are primary goals in conveying to our audience in our presentation."

This comment and others like it point to designers' belief that the Communication phase required a special focus on cybersecurity, for purposes of both clarity and persuasion. While few studies could be found exploring the Communicate phase's specific needs in $\mathrm{HCD}$, clear communication of results between design phases, grounded in the problem frame, has been well-established as foundational to the success of HCD approaches (Roschuni et al., 2015).

\subsection{R2: How do designers' usages of the cards align with the intent of the cards?}

We examine which cards designers selected as most valuable (Fig. 2, Fig. 3a). A Holm-Adjusted pairwise proportion test revealed a significant difference between 'None of the Cards' and other card options across all factors. A significant difference between Card 3 in the diverge design activity and Card 2 in the diverge design activity was also found. No other significant differences were found.

\subsubsection{Result 3.1: The cards were valuable in supporting activities beyond the intended activity.}

We are able to validate that all of the cards, regardless of their intention, proved valuable across all three design activities (Fig. 2). While statistically insignificant, we do see that cards appeared to serve their intention well: Card 1 was most prominent in Frame design activities; Card 2 was most prominent in Diverge design activities (we note that the difference between Card 2 and Card 3 in this design activity was significant, however, suggesting that Card 2 was more prominent than Card 3); and Card 3 was most prominent in Converge design activities. Of these three, we observe that Diverge design activities respond most saliently to the designated intervention to that activity, Card 2.

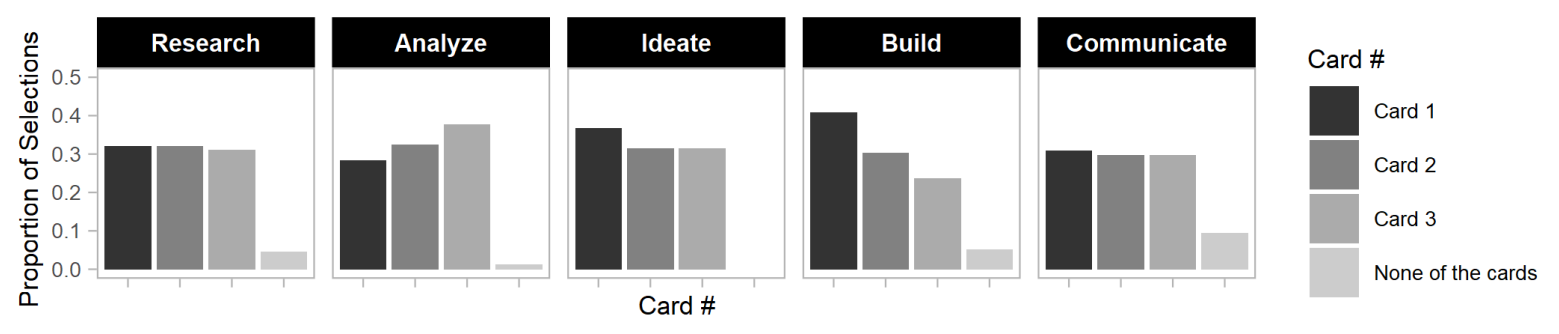

Figure 2. Selection of Cards by Design Phase.

Among the cards, two themes emerged: (1) keeping focused on cybersecurity and (2) treating the cards as a unit. One designer highlighted the first theme in their response, suggesting that the cards helped the team relate their work to the broader issue of cybersecurity:

"The third card helped us re-center our product back [to] cybersecurity, and making sure that we are actually building a product that users feel improve cybersecurity - whether this means that their cards are less vulnerable to hackers, their data not accessible to ad companies, etc."

This serves to, as they write, "re-center" the team's work back to cybersecurity. Surprisingly, the designer is not necessarily referencing psychological distance as a driver of 'forgetting' cybersecurity, but suggesting that the design process itself pushed the team further away from the problem at hand. This builds on earlier findings that design teams struggle to keep their design process focused on usercentered and other project outcomes in later design phases (Rao et al., 2020b), and broader acknowledgment that sustaining empathy for users can be a challenge across the design process (Woodcock et al., 2019). However, we believe that because participants face challenges of psychological distance in working with cybersecurity, teams drift from their focus on the problem domain. This is an important challenge, as designers working in these problem areas are at risk of losing sight of both the problem context and user needs. 
"There is a cyclical nature with all of the cards, allowing us to build and reflect in a very efficient way. Because of this, all of the cards allowed us to frame what we needed to do, as well as create a range of possible improvement options and then select options based on our user feedback."

This designer highlights the second theme in their response, suggesting that design teams engaged with the cards in each phase as a unit, rather than as discrete interventions. This challenges one of the findings in our earlier study, that careful sequencing of card delivery yielded optimal results (Rao et al., 2020a). The 'cyclical nature' of the cards has interesting implications about how designers respond to interventions in psychologically distant and complex problem domains.

\subsubsection{Result 3.2: Card interventions were useful across design phases, with Card 3 delivering more value in the Analyze phase, and Card 1 delivering more value in the Build phase.}

We validate that all of the cards proved valuable across all five design phases. While statistically insignificant, we note that Card 3 delivered the most value among cards in the Analyze phase, and that Card 1 delivered the most value in the Build phase (Fig. 2). We note that the Analyze phase involves teams synthesizing research findings to articulate design opportunities to pursue (Roschuni et al., 2013). This phase is often challenging for design teams, and encompasses what has been described as 'sensemaking' and 'framing' (|Kolko, 2010, 2009). Card 3, inspired by evaluative creativity, was intended to help teams converge during the design phase. Several designers commented on the specific connection between this Card and crafting design opportunities (referred to here as 'how might we statements') and how it helped the team iterate through the work of sensemaking:

"The third question was very helpful in reminding our group to return to the concept of HCD. Often when we attempt to provide solutions to these problems we can get stuck in the research and conversations we have been having internally. Rethinking our framework and consistently renaming is an important step in approaching our problem externally and reminding ourselves of the user's perspective. "

This comment connects Card 3 and the navigation of challenging aspects of the Analyze phase. The Analyze phase is already characterized by a high level of abstraction (Paton and Dorst, 2011); we believe that psychological distance between designers and cybersecurity intensifies this abstraction. Accordingly, teams appear to particularly need support on returning to the core concept of the end user, specifically through an iterative and organizational lens. In the Analyze phase, this is best provided by a card focused on convergent, evaluative activities, which inherently challenges abstraction.

In contrast, the Build phase involves design teams translating abstract concepts into tangible prototypes. A successful transition requires designers to adopt an experimental and learning mindset, while simultaneously detailing concepts in a process of communication (Lauff et al., 2020). Card 1, inspired by analysis creativity, was intended to help teams frame what they needed to achieve in a given design phase. Several designers commented on the connection between Card 1 and the Build phase:

"Card 1 is helpful because during Ideation, our group is distracted from cybersecurity issues and fascinated by various emerging ideas. This card reminds us of our duty."

This comment highlights how the transition to the Build phase requires teams to concretize abstract ideas from the ideate phase. Coughlan et al. calls this 'building to think,' requiring teams to make decisions about aspects of the prototype, but also affording more effective communication (Coughlan et al., 2007). The designer's comment describes a phenomenon similar to Result 2.1 earlier: a distraction from the key goal of cybersecurity. However, the designer particularly addresses the transition from Ideate to Build, and that being 'fascinated by ... emerging ideas' contrasts to the work of the Build phase, which requires them to be aligned with their 'duty.' Furthermore, this comment connects to arguments that higher psychological distance facilitates increased, albeit more abstract, creativity - which perhaps leads students even further away from the core design topic and challenge. In both instances, Card 1 plays a key role in not only helping frame the Build phase, but more broadly helping designers transition from abstract ideas to concrete prototypes. This transition, already in evidence of previous studies of the design process (Rao et al., 2020b), is particularly challenging in problems with high psychological distance, and we believe the noticeable trends in how the cards are a result of this. 


\subsubsection{Result 3.3: The design activities that cards were most valuable for differed from the intent behind the cards during the Research, Analyze and Build phases.}

Examining card preferences across both design phase and design activity (Fig. 3) with a Holm-adjusted pairwise proportion test revealed no significant $(\mathrm{p}<0.05)$ differences between card selection proportions across phases. While not statistically significant, three interesting insights emerged. First, Card 3, developed for evaluative creativity and intended to help teams converge, is used frequently for framing in the Research and Analyze phases (Fig. 3b). One designer reflected on the Research phase:

"Card 3 helped us form a hypothesis and framework of how smart home technology users are at risk for using these types of devices ... [helping] us establish empathy and consider users needs."

As this designer points out, the convergent Card 3 helped teams frame the Research phase. We explain this result by recognizing that with psychologically distant problems, designers in the first stage of the process seek help in concretizing an abstract problem space. This explains why designers might find value in the convergent Card 3 and evaluative creativity in the first step of framing the problem.

Second, Card 1, developed for analysis creativity and intended to help teams frame problems, is used frequently for converging during the Research and Build phases (Fig. 3c). A designer reflected:

"I found card 1 extremely helpful in that it helped us recognize features of our prototype, which we wanted to make more prominent and for us to focus on more."

This suggests that designers combine the step of identifying features (and analogously, end-users) with choosing which among them to pursue. We believe this reflects teams' desire to concretize their work owing to the abstract nature of the project going into each phase: entering the Research phase, the project has just begun, whereas entering the Build phase, the team has just completed the Ideate phase.

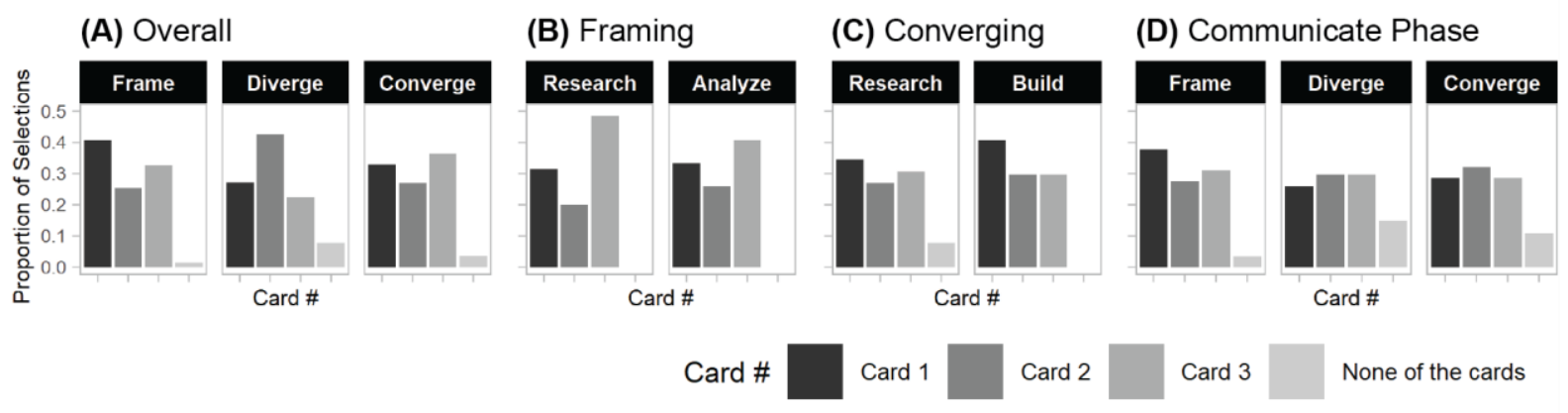

Figure 3. Card selections by (a) Design Activity; (b) Framing Activity (Research and Analyze);

(c) Converging Activity (Research and Build); and (d) all activities (Communicate).

Third, we see that across all activities in the Communicate phase, the Cards were valued similarly. This ties into Result 1.1 as evidenced by one designer's comments, which analogized the Cards to feedback. Feedback is well-known to be helpful throughout the design process, but especially during the Communicate phase, when design teams must synthesize large amounts of information. We anticipate that feedback is particularly helpful in identifying gaps in psychologically distant problems.

\section{IMPLICATIONS FOR DESIGN RESEARCH \& PRACTICE}

This work reveals two immediate implications for design research and practice. First, this work shows that designers find value in the DfC cards simply for 'reminding' them of cybersecurity throughout the design process (Section 4.2.1). While HCD intends to bridge psychological distance, our work suggests additional support is needed to keep designers engaged when the challenge is both complex and distant. For design researchers, this illustrates the need for deeper investigation of the relationship between complex problems, psychological distance, and the classic empathy-building activities of HCD. Similarly, further research into what aspects of psychological distance drives this behavior is essential it. For design practitioners, these findings suggest that design leaders and managers must consider how to sustain design teams' engagement with complex problem domains across the HCD process.

Second, our work suggests that complex and psychologically distant problem spaces may make challenging steps in the HCD process even more difficult (Sections 4.2.2 \& 4.2.3). For design researchers, this invites deeper investigation into how psychological distance between designers and 
problem domains shapes design cognition, and thus, influences design activities and design outcomes. Aspects of design cognition beyond creativity, e.g. abductive thinking, warrant study. These findings suggest that design leaders should ensure that design teams have opportunities to re-connect, or as one student put it, "re-center" their focus, to the problem domain during the design process.

\section{LIMITATIONS}

This study has several important limitations. First, psychological distance was not measured at the outset, conclusion, or longitudinally for individual designers, and some teams and individuals may have had greater or less distance to cybersecurity than others. However, as we argue in Section 2.1, we believe that cybersecurity as a problem domain is characterized by greater psychological distance and our findings accurately represent designer behavior at a high level. Second, we are limited by a small sample size of students and projects. We are thus unable to achieve statistical significance for several claims, nor can we examine how project scope (e.g., some projects were more constrained in scope than others, which were more exploratory) might influence designer behavior. This limitation invites further, larger-scale studies to validate and build on the descriptive results presented here.

\section{CONCLUSIONS}

In this work, we explore how the Design for Cybersecurity (DfC) Cards were used in a project-based design course focused on cybersecurity, which we argue is psychologically distant from many designers. DfC cards were uniformly found useful across the design process. We find that the cards were particularly useful in helping teams "re-center" their focus on cybersecurity as they traverse the design process. We also identify moments in the Research, Analyze, and Build phases where designers especially found value in the cards, which we argue is a result of psychological distance intensifying the abstraction inherent in corresponding design activities. These findings have implications for researching the role of psychological distance in design process, and appropriately managing it.

\section{ACKNOWLEDGMENTS}

This work was partly supported by the Center for Long-Term Cybersecurity (UC-Berkeley).

\section{REFERENCES}

Assal, H., 2018. The Human Dimension of Software Security and Factors Affecting Security Processes. Carleton University.

Bazzano, A.N., Martin, J., Hicks, E., Faughnan, M., Murphy, L., 2017. Human-centred design in global health: A scoping review of applications and contexts. PLoS ONE 12, e0186744. https://doi.org/10.1371/journal.pone.0186744

Bornemann, T., Homburg, C., 2011. Psychological distance and the dual role of price. Journal of Consumer Research 38, 490-504. https://doi.org/10.1086/659874

Coughlan, P., Suri, J.F., Canales, K., 2007. Prototypes as (Design) Tools for Behavioral and Organizational Change: A Design-Based Approach to Help Organizations Change Work Behaviors. The Journal of Applied Behavioral Science 43, 122-134. https://doi.org/10.1177/0021886306297722

Crumpler, W., Lewis, J.A., 2019. Cybersecurity Workforce Gap. Center for Strategic and International Studies (CSIS).

Förster, J., Friedman, R.S., Liberman, N., 2004. Temporal construal effects on abstract and concrete thinking: consequences for insight and creative cognition. Journal of personality and social psychology 87, 177. https://doi.org/10.1037/0022-3514.87.2.177

Hoever, I.J., Van Knippenberg, D., Van Ginkel, W.P., Barkema, H.G., 2012. Fostering team creativity: perspective taking as key to unlocking diversity's potential. Journal of applied psychology 97, 982. https://doi.org/10.1037/a0029159

Howard, T.J., Culley, S.J., Dekoninck, E., 2008. Describing the creative design process by the integration of engineering design and cognitive psychology literature. Design Studies 29, 160-180. https://doi.org/10.1016/j.destud.2008.01.001

Hu, W.-L., Reid, T., 2018. The effects of designers' contextual experience on the ideation process and design outcomes. Journal of Mechanical Design 140. https://doi.org/10.1115/1.4040625

Jia, L., Hirt, E.R., Karpen, S.C., 2009. Lessons from a faraway land: The effect of spatial distance on creative cognition. Journal of Experimental Social Psychology 45, 1127-1131.

https://doi.org/10.1016/j.jesp.2009.05.015 
Kim, E., Jensen, M.B., Poreh, D., Agogino, A.M., 2018. Novice Designer's Lack of Awareness to Cybersecurity and Data Vulnerability in New Concept Development of Mobile Sensing Devices. Presented at the 15th International Design Conference, pp. 2035-2044. https://doi.org/10.21278/idc.2018.0461

Kim, E., Kwon, J., Yoon, J., Agogino, A.M., 2019. Embedding Cybersecurity Into Design Education: Increasing Designers' Awareness of Cybersecurity Throughout the Design Process, in: ASME 2019 International Design Engineering Technical Conferences and Computers and Information in Engineering Conference. American Society of Mechanical Engineers Digital Collection. https://doi.org/10.1115/DETC2019-97720

Kolko, J., 2010. Sensemaking and framing: A theoretical reflection on perspective in design synthesis. Design Research Society.

Kolko, J., 2009. Abductive Thinking and Sensemaking: The Drivers of Design Synthesis. Design Issues 26, 15-28. https://doi.org/10.1162/desi.2010.26.1.15

Lauff, C.A., Knight, D., Kotys-Schwartz, D., Rentschler, M.E., 2020. The role of prototypes in communication between stakeholders. Design Studies 66, 1-34. https://doi.org/10.1016/j.destud.2019.11.007

Liberman, N., Trope, Y., 2008. The psychology of transcending the here and now. Science 322, 1201-1205. https://doi.org/10.1126/science.1161958

Lukowiak, M., Radziszowski, S., Vallino, J., Wood, C., 2014. Cybersecurity education: Bridging the gap between hardware and software domains. ACM Transactions on Computing Education (TOCE) 14, 2. https://doi.org/10.1145/2538029

Norman, D.A., Stappers, P.J., 2015. DesignX: Complex Sociotechnical Systems. She Ji: The Journal of Design, Economics, and Innovation 1, 83-106. https://doi.org/10.1016/j.sheji.2016.01.002

Pahl, S., Bauer, J., 2013. Overcoming the distance: Perspective taking with future humans improves environmental engagement. Environment and Behavior 45, 155-169. https://doi.org/10.1177/0013916511417618

Paton, B., Dorst, K., 2011. Briefing and reframing: A situated practice. Design Studies, Interpreting Design Thinking 32, 573-587. https://doi.org/10.1016/j.destud.2011.07.002

Polman, E., Emich, K.J., 2011. Decisions for others are more creative than decisions for the self. Personality and Social Psychology Bulletin 37, 492-501. https://doi.org/10.1177/0146167211398362

Postma, C.E., Zwartkruis-Pelgrim, E., Daemen, E., Du, J., 2012. Challenges of doing empathic design: Experiences from industry. International journal of design 6.

Rao, V., Kim, E., Jung, H.J., Goucher-Lambert, K., Agogino, A., 2020a. Design for Cybersecurity (DfC) Cards: A Creativity-Based Approach to Support Designers' Consideration of Cybersecurity, in: Proceedings of the 9th International Conference on Design, Computing, and Cognition DCC'20. Presented at the Design, Computing, and Cognition 2020, Atlanta, GA.

Rao, V., Kim, E., Kwon, J., Agogino, A., Goucher-Lambert, K., 2020b. Method selection in human-centered design teams: an examination of decision-making strategies, in: ASME 2020 International Design Engineering Technical Conferences and Computers and Information in Engineering Conference. St. Louis, MO. https://doi.org/10.1115/DETC2020-22669

Roschuni, C., Agogino, A.M., Beckman, S.L., 2011. The DesignExchange: Supporting the Design Community of Practice, in: DS 68-8: Proceedings of the 18th International Conference on Engineering Design (ICED 11), Impacting Society through Engineering Design, Vol. 8: Design Education, Lyngby/Copenhagen, Denmark, 15.-19.08.2011.

Roschuni, C., Goodman, E., Agogino, A.M., 2013. Communicating actionable user research for human-centered design. AI EDAM 27, 143-154. https://doi.org/10.1017/S0890060413000048

Roschuni, C., Kramer, J., Zhang, Q., Zakskorn, L., Agogino, A., 2015. Design Talking: An Ontology of Design Methods to Support a Common Language of Design, in: Proceedings of the International Conference on Engineering Design. Presented at the International Conference on Engineering Design ICED15.

Schuetz, S.W., Lowry, P.B., Pienta, D.A., Thatcher, J.B., 2015. Improving the design of information security messages by leveraging the effects of temporal distance and argument nature.

Schwartz, S., Ross, A., Carmody, S., Chase, P., Coley, S.C., Connolly, J., Petrozzino, C., Zuk, M., 2018. The Evolving State of Medical Device Cybersecurity. Biomedical Instrumentation \& Technology 52, 103-111. https://doi.org/10.2345/0899-8205-52.2.103

Spence, A., Poortinga, W., Pidgeon, N., 2012. The psychological distance of climate change. Risk Analysis: An International Journal 32, 957-972. https://doi.org/10.1111/j.1539-6924.2011.01695.x

Trope, Y., Liberman, N., 2010. Construal-level theory of psychological distance. Psychological review 117, 440.

Tweneboah-Koduah, S., Skouby, K.E., Tadayoni, R., 2017. Cyber Security Threats to IoT Applications and Service Domains. Wireless Pers Commun 95, 169-185. https://doi.org/10.1007/s11277-017-4434-6

Woodcock, A., McDonagh, D., Magee, P., Ball, T., Iqbal, S., 2019. Expanding Horizons: Engaging Students with Empathic Thinking, in: DS 95: Proceedings of the 21st International Conference on Engineering and Product Design Education (E\&PDE 2019). University of Strathclyde, Glasgow. https://doi.org/10.35199/epde2019.49 